\title{
Riqueza de formigas de solo na praia da Pedreira, Parque Estadual de Itapuã, Viamão, RS, Brasil
}

\author{
Elena Diehl ${ }^{1,2,3}$, Franciele Sacchett ${ }^{2} \&$ Emília Zoppas de Albuquerque ${ }^{2}$
}

\begin{abstract}
${ }^{1}$ Laboratório de Insetos Sociais, Universidade do Vale do Rio dos Sinos. Caixa Postal 275, 93001-970 São Leopoldo-RS, Brasil.
${ }^{2}$ Programa de Pós-Graduação em Biologia: Diversidade e Manejo de Vida Silvestre, Universidade do Vale do Rio dos Sinos. Caixa Postal 275, 93001-970 São Leopoldo-RS, Brasil.

${ }^{3}$ Pesquisadora CNPq. Autor correspondente. ediehl@unisinos.br
\end{abstract}

\begin{abstract}
Richness of ground-dwelling ants in the Praia da Pedreira, Parque Estadual de Itapuã, Viamão, RS, Brazil. Aiming to improve the knowledge on the Brazilian biodiversity, especially the ant fauna of Rio Grande do Sul State (Southern Brazil), this survey was conducted in the Praia da Pedreira, a site of Intensive Use of the Parque Estadual de Itapuã. Ground-dwelling ant species were surveyed for three environments in the beach (native forest, rock bar and sand bar), during 12 months (April/2000 - March/2001). Collections resulted in 60 species belonging to 24 genera, 18 tribes and eight subfamilies (Dolichoderinae, Ecitoninae, Ectatomminae, Formicinae, Heteroponerinae, Myrmicinae, Ponerinae and Pseudomyrmecinae). According to the first order jackknife estimator, the native forest area presented the greatest richness $\left(\mathrm{S}_{\mathrm{sst}}=37.5\right)$, followed by rock bar $\left(\mathrm{S}_{\mathrm{est}}=8.9\right)$ and sand bar $\left(\mathrm{S}_{\mathrm{esc}}=5.9\right)$. Only Acromyrmex laticeps, Crematogaster sp. and Solenopsis invicta were common to all three environments. This paper presents the first record of Pachycondyla crenata and Pachycondyla laevigata (Ponerinae) occurrence in the Rio Grande do Sul State.
\end{abstract}

KEYWORDS. Biodiversity, epygeous ant fauna, Formicidae, Insecta.

RESUMO. Riqueza de formigas de solo na praia da Pedreira, Parque Estadual de Itapuã, Viamão, RS, Brasil. Neste trabalho são listadas as espécies de formigas de solo encontradas em três ambientes (mata nativa, barreira pedregosa e areias da orla) na praia da Pedreira, localizada na Zona de Uso Intensivo do Parque Estadual de Itapuã, município de Viamão, RS. No total, foram identificadas 60 espécies representantes de 24 gêneros, 18 tribos e oito subfamílias (Dolichoderinae, Ecitoninae, Ectatomminae, Formicinae, Heteroponerinae, Myrmicinae, Ponerinae e Pseudomyrmecinae). Dos três ambientes amostrados, de acordo com o estimador de riqueza jackknife de primeira ordem, a mata nativa apresentou a maior riqueza $\left(\mathrm{S}_{\text {est }}=37,5\right)$, seguindo-se a barreira pedregosa $\left(\mathrm{S}_{\text {est }}=8,9\right)$ e as areias da orla $\left(\mathrm{S}_{\text {est }}=5,9\right)$. Apenas Acromyrmex laticeps, Crematogaster sp. e Solenopsis invicta foram comuns aos três ambientes. Neste trabalho é feito o primeiro registro de ocorrência de Pachycondyla crenata e Pachycondyla laevigata (Ponerinae) para o Rio Grande do Sul.

PALAVRAS-CHAVE. Biodiversidade, Formicidae, Insecta, mirmecofauna epigéica.

O impacto negativo que a espécie humana exerce cada vez mais sobre o ambiente, tem levado à necessidade de desenvolver conceitos e estabelecer novos critérios sobre as formas de uso dos recursos ambientais. Um dos conceitos é o de uso sustentável, o qual, segundo Campanhola (2001), necessariamente envolve a interação de três componentes: 0 biológico, o econômico e o social, sendo que cada um destes possui seu próprio conjunto de metas. Citando apenas um exemplo de meta de cada sistema, ter-se-ia respectivamente: manutenção da diversidade biológica, redução da pobreza e justiça social. Assim, segundo o autor, pode ser considerado como um componente do desenvolvimento sustentável qualquer atividade econômica que aumente o bem-estar social, desde que acompanhada pela máxima conservação dos recursos naturais e pela menor degradação ambiental possível dentro das limitações impostas pela ordem sócio-econômicatecnológica. Dentro desta perspectiva poderiam ser enquadradas e justificadas a criação, a manutenção e a ampliação das unidades de conservação, reservas, parques e jardins, sejam federais, estaduais, municipais ou privadas.

No Rio Grande do Sul, o Parque Estadual de Itapuã (PEI) é uma Unidade de Conservação, que objetiva a preservação, a restauração dos recursos naturais e a sua utilização racional e sustentável pela sociedade. Compreende uma grande diversidade de ambientes, tais como banhados, campos úmidos e secos, campos rupestres e matas, dunas, lagoas e restingas, que abrigam flora e fauna significativas. A praia da Pedreira está localizada na Zona de Uso Intensivo do PEI, estando constituída por áreas em grande parte ainda em condições naturais, mas já apresentando alterações decorrentes da atividade humana. A praia da Pedreira engloba, especialmente, áreas típicas das margens do Guaíba e da laguna dos Patos, estando constituída, na sua orla, por areias e rochas, seguida por matas, ainda com presença de muitas figueiras (Ficus spp.) (Plano de Manejo Parque Estadual de Itapuã 1997).

Para subsidiar planos de conservação e/ou de manejo dos ecossistemas e dos ambientes sob proteção ambiental, há necessidade de se utilizar organismos que possam ser indicadores da biodiversidade e das condições ambientais (Majer 1983). Quando esta escolha recai sobre aves e mamíferos, apesar destes não poderem ser considerados os melhores indicadores, têm a seu favor, em especial, o fato de muitas das suas espécies, por serem carismáticas, atraírem favoravelmente a opinião pública (Agosti et al. 2000). Por outro 
lado, a maioria dos diversos grupos de invertebrados, além de exigirem muitos especialistas para sua identificação (Majer 1983), não atraem a atenção da mídia popular.

Um organismo para poder ser utilizado com eficiência como indicador das condições de preservação, degradação ou de recuperação ambiental deve possuir atributos particulares, tais como: riqueza de espécies local e global altas, facilidades para serem amostradas padronizadamente, possibilidade de identificar as espécies ou de separar em morfo-espécies, importância ecológica e respostas rápidas aos estresses ambientais. Dentre os invertebrados terrestres, as formigas por possuírem estes atributos, bem como pelo fato de terem sua diversidade relacionada com vários outros componentes bióticos, são boas indicadoras do estado de conservação dos ecossistemas terrestres (Majer 1983; Andersen 1997; Silva \& Brandão 1999; Alonso 2000).

Visando contribuir ao conhecimento da biodiversidade regional, mas também obter dados que possam subsidiar futuros planos de manejo do PEI, foi realizado um inventário das espécies de formigas em três ambientes da praia da Pedreira.

\section{MATERIAL E MÉTODOS}

O estudo mirmecofaunístico foi realizado durante 12 meses (abril/2000 - março/ 2001), na praia da Pedreira, Parque Estadual de Itapuã - PEI - (30²7'S; 5050'W), no município de Viamão, RS. O clima local é subtropical úmido, sem estação seca, com temperatura média anual de $17,5^{\circ} \mathrm{C}$. A precipitação média anual varia de 1.000 a 1.300 mm (Plano de Manejo Parque Estadual de Itapuã 1997).

As coletas de formigas de solo foram feitas em três ambientes da praia da Pedreira que diferem especialmente na estrutura vegetacional: 1) mata nativa com estrato superior descontínuo, seguido por um estrato arbóreo contínuo formado por árvores não muito altas e arvoretas, e pelo estrato arbustivo-herbáceo, rico em folhiço; 2) barreira pedregosa, onde o granito encontra-se recoberto por musgos, pteridófitas, cactáceas, bromeliáceas, compostas e verbenáceas, compondo uma vegetação tipicamente rupestre; 3) ambiente arenoso e seco da orla, caracterizado pela presença de algumas gramíneas, raras ciperáceas e asteráceas.

$\mathrm{Na}$ mata nativa, barreira pedregosa e nas areias da orla foram traçados dois transectos de $150 \mathrm{~m}$, afastados entre si 20 $\mathrm{m}$, com iscas de sardinha em óleo vegetal distribuídas a cada $10 \mathrm{~m}$, totalizando 30 iscas por ambiente. As iscas foram recolhidas após uma hora da sua exposição. Na mata nativa ainda foram recolhidas dez amostras de $1 \mathrm{~m}^{2}$ de folhiço, que foram triadas em laboratório e, as formigas encontradas, coletadas. Nos três ambientes, também foram feitas coletas diretas, com esforço amostral de uma hora/ambiente. Todo o material coletado foi transferido para vidros individuais com álcool $70 \%$ e os dados da coleta.

Posteriormente, os espécimes foram montados em alfinetes entomológicos e identificados em nível de gênero de acordo com Bolton (1994). As espécies e morfo-espécies foram separadas por comparação com os exemplares da Coleção de
Formicidae do Laboratório de Insetos Sociais da UNISINOS, onde o material testemunho encontra-se depositado. Quando necessário, foram consultados os catálogos de Kempf(1972), Brandão (1991) e de Bolton (1995, 2003).

A riqueza observada $\left(\mathrm{S}_{\mathrm{obs}}\right)$ foi obtida pelo número absoluto de espécies de formigas coletadas por todos os métodos em cada ambiente. A abundância relativa foi calculada com base na freqüência de ocorrência das espécies nas iscas e amostras de folhiço (e não no número de indivíduos) dividido pelo número total de iscas e amostras de serapilheira. Este procedimento é mais adequado para comparações interespecíficas, pois os cálculos não sofrem os efeitos do tamanho das colônias, nem do comportamento de recrutamento das operárias (Romero \& Jaffé 1989), o que levaria a superestimar aquelas espécies com sistemas de recrutamento mais eficiente e/ou aquelas cujas colônias estão mais próximas das iscas (Leal \& Lopes 1992). Para a projeção de riqueza, ou riqueza estimada $\left(\mathrm{S}_{\text {est }}\right)$, foi usado o estimador de riqueza jackknife de primeira ordem, pelo programa EstimateS, versão 5.0.1 (Colwell 1997) utilizando para os ambientes mata nativa, barreira pedregosa e areias da orla apenas os dados obtidos com as iscas de sardinha. Para a análise da similaridade entre as comunidades dos três ambientes foi feita análise de cluster pela distância euclidiana (Wilkinson 2000).

\section{RESULTADOS}

Considerando as formigas coletadas por todos os métodos e no total dos três ambientes da praia da Pedreira, foram identificadas 60 espécies, distribuídas em 24 gêneros, 18 tribos e oito subfamílias (Dolichoderinae, Ecitoninae, Ectatomminae, Formicinae, Heteroponerinae, Myrmicinae, Ponerinae e Pseudomyrmecinae). Destas, representantes de sete subfamílias foram encontradas na mata nativa, quatro na barreira pedregosa e três nas areias da orla (Tabela I). As curvas obtidas (Figura 1) para as estimativas de riqueza nos três ambientes, mostram que nas areias da orla e na barreira pedregosa o número de iscas foi suficiente para determinar a riqueza pois as curvas estabilizaram-se, praticamente, a partir da sexta e nona iscas. No entanto, para a mata nativa, ainda na $30^{\mathrm{a}}$ isca a curva mostrava uma tendência de registrar maior riqueza de espécies com maior esforço amostral.

Do total das 60 espécies registradas na praia da Pedreira, 16 foram obtidas apenas por coleta manual (Tabela I). Dos três ambientes amostrados (Tabela II), e levando em consideração também as espécies coletadas manualmente e no folhiço quando presente, a mata nativa apresentou a maior riqueza observada $\left(\mathrm{S}_{\mathrm{obs}}=53\right)$, seguindo-se a barreira pedregosa $\left(\mathrm{S}_{\mathrm{obs}}=12\right)$ e as areias da orla $\left(\mathrm{S}_{\mathrm{obs}}=8\right)$.

Para os gêneros Pheidole (Myrmicinae) e Camponotus (Formicinae), foram registradas as maiores riquezas específicas, respectivamente, 15 e oito espécies (Tabela I).

Da tribo Attini, foram encontradas cinco espécies do gênero Acromyrmex: A. ambiguus Emery, 1887, A. laticeps Emery, 1905, A. lundi (Guérin, 1838), A. striatus (Roger, 1863) e A. subterraneus Forel, 1893, além de um expressivo número de 
Tabela I - Formigas coletadas em três ambientes da praia da Pedreira, Parque Estadual de Itapuã, Viamão, RS

\begin{tabular}{|c|c|c|c|c|c|}
\hline Subfamília & Tribo & Espécie & Areias da Orla & Barreira Pedregosa & MataNativa \\
\hline \multirow[t]{4}{*}{ Dolichoderinae } & \multirow[t]{4}{*}{ Dolichoderini } & Dorymyrmex sp. & $\mathrm{x}^{*}$ & - & - \\
\hline & & Dorymyrmex sp.1 & $\mathrm{x}$ & - & - \\
\hline & & Linepithema sp. & - & - & $\mathrm{x}$ \\
\hline & & Linepithema sp.1 & - & - & $\mathrm{x}$ \\
\hline Ecitoninae & Ecitonini & Labidus coecus (Latreille, 1802) & - & - & $\mathrm{x}$ \\
\hline \multirow[t]{2}{*}{ Ectatomminae } & \multirow[t]{2}{*}{ Ectatommini } & Ectatomma permagnum Forel 1908 & - & - & $\mathrm{x}$ \\
\hline & & Ectatomma sp. 1 & - & - & $\mathrm{x}$ \\
\hline \multirow[t]{12}{*}{ Formicinae } & \multirow[t]{8}{*}{ Camponotini } & Camponotus mus Roger, 1863 & $\mathrm{x}$ & $\mathrm{x}$ & - \\
\hline & & Camponotus rufipes (Fabricius, 1775) & - & $x^{*}$ & $\mathrm{x}$ \\
\hline & & Camponotus sp. & - & - & $\mathrm{x}$ \\
\hline & & Camponotus sp.1 & & - & $\mathrm{x}^{*}$ \\
\hline & & Camponotus sp.3 & - & $\mathrm{x}$ & $\mathrm{x}^{*}$ \\
\hline & & Camponotus sp.5 & - & $\mathrm{x}$ & $\mathrm{x}$ \\
\hline & & Camponotus sp.6 & - & - & $\mathrm{x}$ \\
\hline & & Camponotus sp.12 & - & - & $\mathrm{x}$ \\
\hline & \multirow[t]{3}{*}{ Brachymyrmecini } & Brachymyrmex sp & - & - & $\mathrm{x}$ \\
\hline & & Brachymyrmex sp.1 & - & $\mathrm{x}^{*}$ & - \\
\hline & & Brachymyrmex sp.2 & $\mathrm{x}$ & - & - \\
\hline & Lasiini & Paratrechina sp. & - & - & $\mathrm{x}$ \\
\hline \multirow[t]{2}{*}{ Heteroponerinae } & \multirow[t]{2}{*}{ Heteroponerini } & Heteroponera mayri Kempf, 1962 & - & - & $\mathrm{x}$ \\
\hline & & Heteroponera sp. & - & - & $\mathrm{x}$ \\
\hline \multirow[t]{31}{*}{ Myrmicinae } & \multirow[t]{7}{*}{ Attini } & Acromyrmex ambiguus (Emery, 1888) & $x^{*}$ & - & - \\
\hline & & Acromyrmex laticeps Emery, 1905 & $\mathrm{x} *$ & $\mathrm{x}$ & $\mathrm{x}$ \\
\hline & & Acromyrmex lundi (Guérin, 1838) & - & - & $\mathrm{x}^{*}$ \\
\hline & & Acromyrmex striatus (Roger, 1863) & - & - & $x^{*}$ \\
\hline & & Acromyrmex subterraneus Forel, 1893 & & & $\mathrm{x} *$ \\
\hline & & Cурһотугтех sp. & - & - & $x^{*}$ \\
\hline & & Trachymyrmex sp. & - & - & $x^{*}$ \\
\hline & Blepharidattini & Wasmannia sp. & - & - & $\mathrm{x}$ \\
\hline & \multirow{2}{*}{ Cephalotini } & Procryptocerus sp. & - & - & $x^{*}$ \\
\hline & & Cephalotes depressus (Klug, 1824) & - & $\mathrm{x}^{*}$ & $x^{*}$ \\
\hline & \multirow{2}{*}{ Crematogastrini } & Crematogaster sp. & $\mathrm{x}$ & $\mathrm{x}$ & $\mathrm{x}$ \\
\hline & & Crematogaster sp. 1 & - & - & $x^{*}$ \\
\hline & Dacetini & Strumigenys sp. & - & - & $\mathrm{x}$ \\
\hline & Myrmicini & Hylomyrma sp. 1 & - & $\mathrm{x}$ & $x^{*}$ \\
\hline & \multirow[t]{15}{*}{ Pheidolini } & Pheidole fallax Mayr, 1870 & - & - & $\mathrm{x}$ \\
\hline & & Pheidole fimbriata Roger, 1863 & - & - & $\mathrm{x}$ \\
\hline & & Pheidole sp. & & & $\mathrm{x}$ \\
\hline & & Pheidole sp.1 & - & - & $\mathrm{x}$ \\
\hline & & Pheidole sp.2 & - & - & $\mathrm{x}$ \\
\hline & & Pheidole sp. 3 & - & - & $\mathrm{x}$ \\
\hline & & Pheidole sp. 5 & - & - & $\mathrm{x}$ \\
\hline & & Pheidole sp.6 & - & - & $\mathrm{x}$ \\
\hline & & Pheidole sp.7 & - & - & $\mathrm{x}$ \\
\hline & & Pheidole sp.9 & - & - & $\mathrm{x}$ \\
\hline & & Pheidole sp.10 & - & - & $x^{*}$ \\
\hline & & Pheidole sp.11 & & & $x^{*}$ \\
\hline & & Pheidole sp.13 & - & - & $x^{*}$ \\
\hline & & Pheidole sp.15 & - & - & $\mathrm{x}$ \\
\hline & & Pheidole sp.17 & - & - & $\mathrm{x}$ \\
\hline & \multirow[t]{2}{*}{ Solenopsidini } & Solenopsis invicta Buren, 1972 & $x^{*}$ & $\mathrm{x}$ & $\mathrm{x} *$ \\
\hline & & Solenopsis sp. & - & - & $\mathrm{x}$ \\
\hline Ponerinae & Odontomachini & Anochetus sp. & - & - & $\mathrm{x}$ \\
\hline & & Odontomachus sp. & - & - & $\mathrm{x}$ \\
\hline & Ponerini & Hypoponera foreli (Mayr, 1887) & - & - & $\mathrm{x}$ \\
\hline & & Hypoponera trigona (Mayr, 1887) & - & - & $\mathrm{x}$ \\
\hline & & Pachycondyla crenata (Roger, 1861) & - & - & $\mathrm{x}$ \\
\hline & & Pachycondyla laevigata (F. Smith, 1858) & - & - & $\mathrm{x}^{*}$ \\
\hline & & Pachycondyla striata F. Smith, 1858 & - & $x^{*}$ & $\mathrm{x}$ \\
\hline Pseudomyrmecinae & Pseudomyrmecini & Pseudomyrmex gracilis (Fabricius, 1804) & - & $x^{*}$ & - \\
\hline 8 & 18 & 60 & 8 & 12 & 53 \\
\hline
\end{tabular}

*Espécies obtidas por coleta direta 
colônias de Cyphomyrmex sp. e de alguns ninhos de Trachymyrmex sp. (Tabela I). Paralelamente, distante cerca de $500 \mathrm{~m}$ da praia da Pedreira foi observado um ninho de Atta sexdens piriventris Santschi, 1919 mas que, por estar fora das áreas estipuladas para coleta, não foi considerada para os cálculos de riqueza.

Da subfamília Ponerinae foram coletadas três espécies do gênero Pachycondyla, sendo que de Brachymymex (Formicinae) também foram obtidas três espécies. Para os demais gêneros, foram encontradas apenas uma ou duas espécies (Tabela I).

Do total de espécies coletadas na praia da Pedreira, três $(0,5 \%)$ foram comuns aos ambientes amostrados: A. laticeps, Crematogaster sp. e Solenopsis invicta Buren, 1972.

Comparando as três comunidades pela análise de cluster, as areias da orla e a barreira pedregosa formaram um agrupamento. A mata nativa, por apresentar a maior riqueza e abundância de espécies, ficou separada e bastante distanciada daquele grupo (Figura 2).

\section{DISCUSSÃO}

Tendo em vista as grandes diferenças da complexidade vegetacional entre os ambientes amostrados na praia da Pedreira, especialmente na mata nativa e, portanto, a disponibilidade de alimento e de locais para nidificação (Majer 1983; Benson \& Harada 1988; Matos et al. 1994; Alonso 2000; Brown 2000), os resultados obtidos eram esperados. Do total de espécies encontradas na praia da Pedreira, apenas $0,5 \%$ foi comum aos ambientes amostrados, o que além de indicar reduzida conectividade entre eles e as grandes diferenças nos recursos disponíveis, mostra a baixa migração de formigas entre os três ambientes.

Os gêneros Pheidole (Myrmicinae) e Camponotus (Formicinae) apresentaram a maior riqueza específica o que está de acordo com Wilson (1976), além destes dois gêneros apresentarem ampla distribuição geográfica e grande diversidade de adaptações ecológicas (Hölldobler \& Wilson

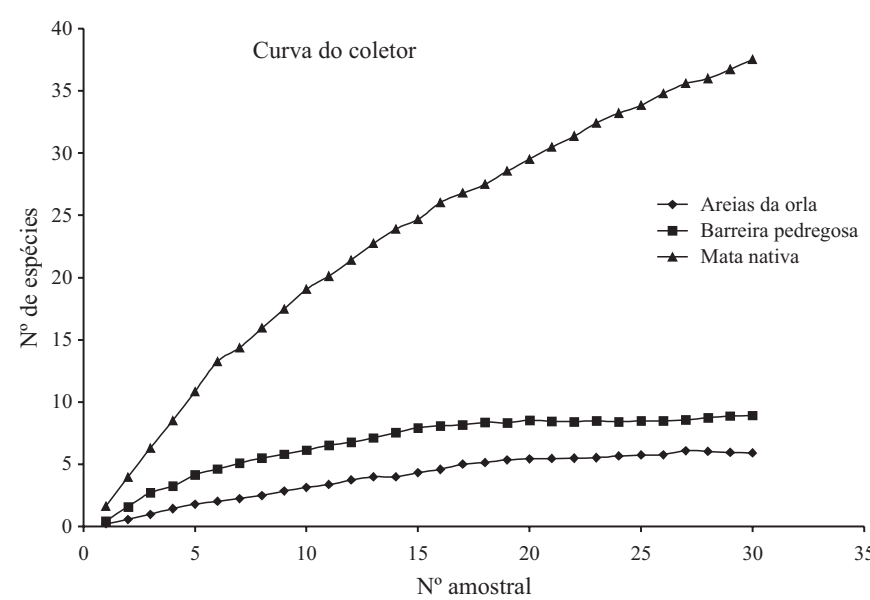

Fig. 1 - Riqueza estimada de espécies de formigas em três ambientes da praia da Pedreira, Parque Estadual de Itapuã, Viamão, RS.
Tabela II - Riquezas, observada $\left(\mathrm{S}_{\mathrm{obs}}\right)$ e estimada $\left(\mathrm{S}_{\text {est }}\right)$, de formigas em três ambientes da praia da Pedreira, Parque Estadual de Itapuã, Viamão, RS.

\begin{tabular}{ccc}
\hline Ambiente & $\mathrm{S}_{\text {obs }}{ }^{(*)}$ & $\mathrm{S}_{\text {est }}$ \\
\hline Mata nativa & $37(53)$ & 37,5 \\
Barreira pedregosa & $7(12)$ & 8,9 \\
Areias da orla & $4(8)$ & 5,9
\end{tabular}

Valores entre parênteses incluem dados das iscas de sardinha, coleta direta e do folhiço (no caso da mata nativa).

1990; Bolton 1994). Resultados similares foram encontrados em ambientes de restinga e de mata de restinga no morro da Grota, também no PEI (Sacchett \& Diehl 2004), bem como por diversos outros autores em outras regiões (Benson \& Harada 1988; Leal \& Lopes 1992; Marinho et al. 2002; Hameister et al. 2003; Fonseca \& Diehl 2004).

Pertencentes à subfamília Ponerinae, foram encontradas três espécies do gênero Pachycondyla, das quais $P$. crenata (Roger, 1861) e P. laevigata (Smith, F., 1858) não estavam citadas nos catálogos de Kempf (1972) e de Brandão (1991), como de ocorrência para o Rio Grande do Sul. Desta forma, este é o primeiro registro destas duas espécies para o estado.

No morro da Grota, também no PEI, Sacchett \& Diehl (2004) registraram riqueza total $\left(\mathrm{S}_{\mathrm{obs}}=61\right)$ similar de espécies de formigas a da praia da Pedreira. Paralelamente, ela foi similar à riqueza observada $\left(\mathrm{S}_{\mathrm{obs}}=51\right)$ para o Parque das Guaritas, área ainda muito bem preservada na Bacia do Camaquã, na região Sudoeste do estado (Diehl et al. 2004). No entanto, a riqueza total na praia da Pedreira foi muito superior à encontrada $\left(\mathrm{S}_{\mathrm{obs}}=33\right)$ no morro de Itapeva em Torres (litoral norte do estado), caracterizado por restingas e dunas móveis (Hameister et al.2003), mas já com grande atividade humana, especialmente turística, bem como à registrada $\left(\mathrm{S}_{\mathrm{obs}}=20\right)$ em uma área suburbana do município de São Leopoldo na região da Encosta Inferior do Nordeste do Rio Grande do Sul por Haubert et al. (1998).

Os resultados obtidos mostram para a praia da Pedreira uma grande riqueza de espécies de formigas, possivelmente resultante da disponibilidade e variedade de locais de nidificação e de recursos alimentares fornecidos pela alta diversidade, vegetal e animal existentes e que, apesar da ação antrópica local, ainda são consideravelmente grandes. No entanto, deve ser destacado o reduzido número de espécies comuns aos três ambientes, apenas $0,5 \%$ da riqueza observada, o que indica uma baixa conectividade entre eles, assim como também pode estar indicando um efeito disruptivo local pela intensa atividade humana ocorrida até há bem pouco tempo. Por sua vez, a ocorrência de Labidus coecus (Latreille, 1802) na mata nativa da praia da Pedreira, sugere fortemente o bom estado de conservação (vide Brown Jr. 2000) não só desta zona, mas do Parque como um todo, visto que esta espécie de formiga legionária necessita de grandes extensões ao longo das quais desloca-se constantemente em busca de alimento. Cabe destacar que Sacchett \& Diehl (2004) também relataram a ocorrência desta espécie no morro da Grota (PEI). 


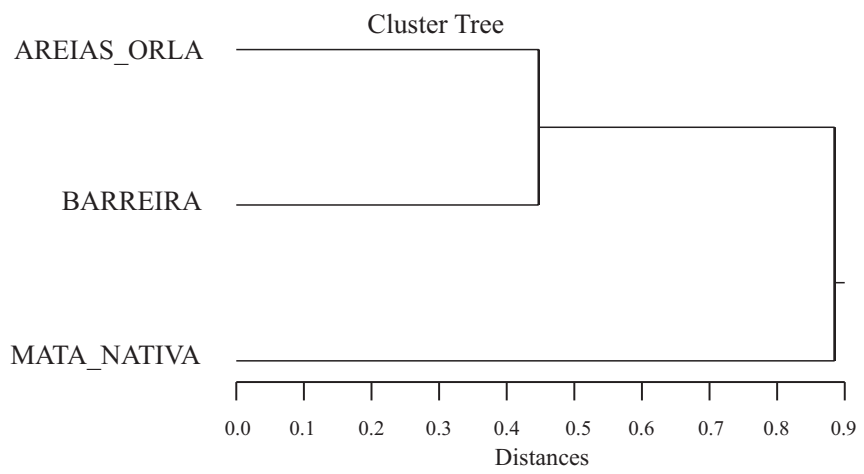

Fig. 2 - Similaridade entre as comunidades de formigas, baseada na distância Euclidiana, dos três ambientes da praia da Pedreira, Parque Estadual de Itapuã, Viamão, RS.

Segundo Campanhola (2001), para a manutenção da diversidade biológica, redução da pobreza e para que ocorra justiça social, devem ser considerados como componentes do desenvolvimento sustentável as atividades econômicas que aumentam o bem-estar social, quando acompanhadas pela máxima conservação dos recursos naturais locais e pela menor degradação ambiental possível dentro das limitações impostas pela ordem sócio-econômica-tecnológica vigente. Estes componentes deverão ser considerados quando da reavaliação das Unidades de Conservação, dentre as quais, no Rio Grande do Sul destaca-se o Parque Estadual de Itapuã, situado em plena região metropolitana e que detém além de inúmeros locais para lazer, importantes ambientes para preservação e conservação. Os resultados obtidos neste trabalho no PEI mostram que apesar da praia da Pedreira ser Zona de Uso Intensivo e, portanto, sofrer intensa ação antrópica, destacando-se a recreação, ainda conserva uma mirmecofauna bastante rica, especialmente quando se considera seu remanescente de mata nativa. Pelo fato das formigas ocuparem uma posição no topo da cadeia trófica (Majer 1983; Hölldobler \& Wilson 1990; Andersen 1997; Alonso 2000), a sua riqueza e abundância certamente reflete a grande diversidade da flora e fauna locais existentes.

Agradecimentos. À Secretaria de Meio Ambiente do Estado do Rio Grande do Sul a autorização para coletas e estudos no Parque Estadual de Itapuã. Ao CNPq, FAPERGS e UNISINOS as bolsas e auxílios concedidos. F.S. foi bolsista PIBIC/CNPq e E.Z.A, bolsista IC/FAPERGS durante o projeto. Agradecimentos especiais ao Dr. J.H.C. Delabie do Laboratório de Mirmecologia da CEPLAC (Itabuna, BA) e ao Dr. Fernando J. Zara da UNESP (Jaboticabal, SP) pela identificação de algumas formigas.

\section{REFERÊNCIAS}

Agosti, D.; J. D. Majer; L. E. Alonso \& T. R. Schultz (eds.). 2000. Preface. Ants: Standard methods for measuring and monitoring biodiversity. Washington, Smithsonian Institution Press, $280 \mathrm{p}$.

Alonso, L. E. 2000. Ants as indicators of diversity, p. 80-88. In: D. Agosti, J. D. Majer, L. E. Alonso \& T. R. Schultz (eds.). Ants:
Standard methods for measuring and monitoring biodiversity. Washington, Smithsonian Institution Press, 280 p.

Andersen, A. N. 1997. Using ants as bioindicators: Multiscale issues in ant community ecology. Conservation Ecology 1: 8. http:// www.consecol.org/vol1/iss1/art8.

Benson, W. \& A. Y. Harada. 1988. Local diversity of tropical and temperate ant faunas (Hymenoptera, Formicidae). Acta Amazonica 18: 275-289.

Bolton, B. 1994. Identification guide to the ant genera of the world. Cambridge, Harvard University Press, $222 \mathrm{p}$.

Bolton, B. 1995. A new general catalogue of the ants of the world. Cambridge, Harvard University Press, $504 \mathrm{p}$.

Bolton, B. 2003. Synopsis and classification of Formicidae. Florida, The American Entomological Institute, 370 p.

Brandão, C. R. F. 1991. Adendos ao catálogo abreviado das formigas da região neotropical (Hymenoptera: Formicidae). Revista Brasileira de Entomologia 35: 319-412.

Brown Jr., W. L. 2000. Diversity of ants, p. 45-79. In: D. Agosti, J. D. Majer, L. E. Alonso \& T. R. Schultz (eds.). Ants: Standard methods for measuring and monitoring biodiversity. Washington, Smithsonian Institution Press, $280 \mathrm{p}$.

Campanhola, C. 2001. Processos e pressões antrópicas que degradam a biodiversidade: estudos de casos, p. 89-91. In: I. Garay \& B. Dias (eds.) Conservação da biodiversidade em ecossistemas tropicais. Petrópolis, Vozes, $432 \mathrm{p}$.

Colwell, R. K. 1997. Estimates: statistical estimation of species richness and shared species from samples. Version 5.0.1. <http:// viceroy.eeb.uconn.edu/ estimate $>$.

Fonseca, R. C. \& E. Diehl. 2004. Riqueza de formigas (Hymenoptera, Formicidae) epigéicas em povoamentos de Eucalyptus spp. (Myrtaceae) de diferentes idades no Rio Grande do Sul, Brasil. Revista Brasileira de Entomologia 48: 95-100.

Hameister, T. M.; Ed. Diehl-Fleig \& E. Diehl. 2003. Comunidades de formigas (Hymenoptera: Formicidae) epígeas no morro de Itapeva, município de Torres, RS. Acta Biologica Leopoldensia 25: $187-$ 195.

Haubert, F.; E. Diehl-Fleig \& A. J. Mayhé-Nunes. 1998. Mirmecofauna de solo no município de São Leopoldo, RS: Levantamento Preliminar. Acta Biologica Leopoldensia 20: 103-108.

Hölldobler, B. \& E. O. Wilson. 1990. The Ants. Cambridge, The Belknap Press of Harvard University, 732 p.

Kempf, W. W. 1972. Catálogo abreviado de formigas neotropicais (Hymenoptera, Formicidae). Studia Entomologica 15: 1-344.

Leal, I. R. \& B. C. Lopes. 1992. Estrutura das comunidades de formigas (Hymenoptera: Formicidae) de solo e vegetação no Morro da Lagoa da Conceição, Ilha de Santa Catarina, SC. Biotemas 5: 107-122.

Majer, J. D. 1983. Ants: Bio-indicators of minesite rehabilitation, landuse, and land conservation. Environmental Management 7: 375383.

Marinho, C. G. S.; R. Zanetti; J. H. C. Delabie; M. N. Schlindwein \& L. S. Ramos. 2002. Diversidade de formigas (Hymenoptera: Formicidae) da serapilheira em eucaliptais (Myrtaceae) e área de cerrado de Minas Gerais. Neotropical Entomology 31: 187-195.

Matos, J. Z.; C. N. Yamanaka; T. T. Castellani \& B. C. Lopes. 1994. Comparação da fauna de formigas de solo em áreas de plantio de Pinus elliottii, com diferentes graus de complexidade estrutural (Florianópolis, SC). Biotemas 7: 57-64.

Plano de Manejo Parque Estadual de Itapuã. 1997. Porto Alegre, Departamento de Recursos Naturais Renováveis, $158 \mathrm{p}$.

Romero, H. \& K. Jaffé. 1989. A comparison of methods for sampling ants (Hymenoptera, Formicidae) in savannas. Biotropica 21: 348 325.

Sacchett, F. \& E. Diehl. 2004. Comunidades de formigas de solo no morro da Grota, Parque Estadual de Itapuã, RS. Acta Biologica Leopoldensia 26: 79-92.

Silva, R. R. da \& C. R. F. Brandão. 1999. Formigas (Hymenoptera: Formicidae) como indicadoras da qualidade ambiental e da biodiversidade de outros invertebrados terrestres. Biotemas 12: $55-73$.

Wilkinson, L. 2000. SYSTAT: the system for statistics. Evaston, Illinois.

Wilson, E. O. 1976. Which are the most prevalent ant genera? Studia Entomologica 19: 187-200.

Recebido em 15.III.2005; aceito em 10.IX.2005 